Separ at e- type scanner and wi deband hi gh- vol tage ampl ifi er for at omic-resol ut i on and hi gh-speed at omi c force mi croscopy

\begin{tabular}{|l|l|}
\hline 著者 & $\begin{array}{l}\text { M yat a Kazuki, Usho Sat oshi, Yanada Sat oshi , } \\
\text { Fur uya Shoj i, Yoshi da Ki yonori, Asakawa } \\
\text { H toshi, Fukuna Takeshi }\end{array}$ \\
\hline 著者別表示 & 宮田 一輝, 淺川 雅, 福間 岡士 \\
\hline $\begin{array}{l}\text { j our nal or } \\
\text { publ i cat i on ti tl e }\end{array}$ & Revi ew of Sci ent i f i c I nst rument s \\
\hline vol une & 84 \\
\hline number & 4 \\
\hline page r ange & 43705 \\
\hline year & 2013 04 01 \\
\hline URL & ht t p: //doi . or g/10. 24517/00008809 \\
\hline
\end{tabular}




\title{
Separate-type scanner and wideband high-voltage amplifier for atomic-resolution and high-speed atomic force microscopy
}

\author{
Kazuki Miyata, ${ }^{1}$ Satoshi Usho, ${ }^{1}$ Satoshi Yamada, ${ }^{1}$ Shoji Furuya,${ }^{1}$ Kiyonori Yoshida,${ }^{1}$ \\ Hitoshi Asakawa, ${ }^{2}$ and Takeshi Fukuma ${ }^{1,2, a)}$ \\ ${ }^{1}$ Division of Electrical Engineering and Computer Science, Kanazawa University, Kakuma-machi, \\ Kanazawa 920-1192, Japan \\ ${ }^{2}$ Bio-AFM Frontier Research Center, Kanazawa University, Kakuma-machi, Kanazawa 920-1192, Japan
}

(Received 22 February 2013; accepted 5 April 2013; published online 23 April 2013)

\begin{abstract}
We have developed a liquid-environment atomic force microscope with a wideband and low-noise scanning system for atomic-scale imaging of dynamic processes at solid/liquid interfaces. The developed scanning system consists of a separate-type scanner and a wideband high-voltage amplifier (HVA). By separating an $X Y$-sample scanner from a $Z$-tip scanner, we have enabled to use a relatively large sample without compromising the high resonance frequency. We compared various cantileverand sample-holding mechanisms by experiments and finite element analyses for optimizing the balance between the usability and frequency response characteristics. We specifically designed the HVA to drive the developed scanners, which enabled to achieve the positioning accuracy of 5.7 and $0.53 \mathrm{pm}$ in the $X Y$ and $Z$ axes, respectively. Such an excellent noise performance allowed us to perform atomic-resolution imaging of mica and calcite in liquid. Furthermore, we demonstrate in situ and atomic-resolution imaging of the calcite crystal growth process in water. ( 2013 AIP Publishing LLC. [http://dx.doi.org/10.1063/1.4802262]
\end{abstract}

\section{INTRODUCTION}

Detailed understanding of interfacial phenomena is often a critical issue in many of the academic and industrial research areas. To achieve this goal, it is ideal to directly image interfacial phenomena with atomic- or molecular-scale resolution. For example, direct imaging of dynamic behavior of proteins should unravel the mechanism of various biological processes. ${ }^{1,2}$ Similarly, direct imaging of dynamic changes in the surface structure should clarify the detailed crystal growth mechanism of various minerals. ${ }^{3,4}$

Atomic force microscopy (AFM $)^{5}$ is one of the nanoscale and non-destructive imaging tools. AFM can be operated in liquid as well as in air and vacuum. In addition, it can be used for imaging of insulating materials as well as conductive ones. Owing to these unique capabilities, AFM has been used for various applications and considered to be suitable for direct imaging of interfacial phenomena with nanoscale resolution.

In situ imaging of dynamic processes requires highspeed operation of AFM. Thus, efforts have been made for improving the AFM operation speed. ${ }^{3,6,7}$ The enhancement of AFM operation speed requires improvement of all the components constituting the tip-sample distance regulation loop. In particular, a scanner is one of the most important components and often determines the maximum imaging speed.

There are two major requirements for driving a scanner at a high speed: high resonance frequency and low cross talk between $X Y$ and $Z$ scanners. To satisfy these requirements, a number of ideas have been proposed. Ando et al. reduced the

a)Electronic mail: fukuma@staff.kanazawa-u.ac.jp cross talk using a counter balance mechanism and enabled high-speed $Z$ feedback regulation. ${ }^{6,8}$ Miles and co-workers avoided the cross talk problem by eliminating the $Z$ feedback mechanism and used a resonant vibration of a quartz oscillator for high-speed $X$ scan. $^{7}$ Hansma et al. used a parallel flexure stage to enhance the resonance frequency of the $X Y$ scanner. ${ }^{9-11}$ Subsequently, the design has been further improved by other researchers. ${ }^{12,13}$ Tabak et al. developed a micrometer-sized $Z$ scanner with an integrated tip for obtaining a high resonance frequency. ${ }^{14}$ They also used an $X Y$ sample scanner that is separated from the $Z$ scanner to achieve low cross talk. Fukuma et al. proposed the inertia balance support mechanism of $Z$ actuator to achieve a high resonance frequency and low cross talk. ${ }^{15}$ More comprehensive review is found in the literature. . $^{8} 16$

Although some of these scanners have already been used for practical studies, there is large room for further improvements especially in the usability and resolution. For example, most of the high-speed scanners require a small sample size, which has limited the applicability of high-speed AFMs. In addition, a sample is typically fixed to a scanner with glue (e.g., expoxy, wax, etc.) to avoid yielding spurious resonances. Thus, we need to wait for the glue to set when we start an experiment or exchange samples. This problem is particularly serious for atomic-scale experiments that require a tip drift rate typically less than $1 \mathrm{~nm} / \mathrm{s}$. To achieve this performance, we often need to leave the fixed sample for more than several hours.

Another issue is the resolution. Simultaneous achievement of high speed and high resolution requires low noise and wideband scanning system including a high voltage amplifier (HVA) and a scanner. So far, high-speed AFM has mostly been used for nanoscale applications. To apply high-speed 
AFM to atomic-scale applications, noise of a HVA should be reduced without deteriorating the bandwidth.

In this study, we have developed a high-speed scanning system with atomic-scale resolution and sufficient usability. To avoid the cross talk, we used separate $X Y$ sample and $Z$ tip scanners. To achieve both high speed and sufficient usability, we have optimized the holding mechanisms for the sample and tip by comparing the results of experiments and simulations. We have also developed a low noise and wideband HVA that is specifically designed to drive the developed scanner. We demonstrate the improved speed and resolution by imaging mica in liquid with atomic resolution. In addition, we present in situ imaging of the growth process of a calcite crystal in water with atomic-scale resolution.

\section{EXPERIMENTAL DETAILS}

Theoretical analyses of the frequency response and vibration modes of the scanners were performed by finite element analysis (FEA) software (COMSOL Multiphysics, COMSOL). The COMSOL models used in this study are included in the supplementary material. ${ }^{17}$ For modeling the scanner bodies made of SS304 or SS430, we used Young's modulus of 197 and $200 \mathrm{GPa}$, Poisson's ratio of 0.3, and density of 7930 and $7750 \mathrm{~kg} / \mathrm{m}^{3}$. For modeling the stack piezoactuators, we used density of $7500 \mathrm{~kg} / \mathrm{m}^{3}$. In addition, we experimentally determined their coupling and elasticity matrices by measuring the displacement and resonance frequency of the driven actuators with and without fixation to a large body.

We measured the frequency response of the developed scanning system by a frequency response analyzer (FRA5097, NF). For detecting the displacement of the $X Y$ scanner, we measured torsional vibration of a cantilever in contact with the surface of the sample stage by the AFM cantilever deflection sensor. For detecting the displacement of the $Z$ scanner, we measured the vertical vibration of a cantilever body attached to the cantilever stage by a heterodyne laser displacement sensor (ST-3761, IWATSU). The output voltage spectral density distribution of the HVA was measured by a fast Fourier transform (FFT) analyzer implemented in the cantilever oscillation controller (OC4, SPECS). The output from the HVA is fed into the FFT analyzer through a $1 \mathrm{kHz}$ highpass filter to remove the dc voltage component.

The AFM imaging was performed by a home-built AFM with a low-noise cantilever deflection sensor. ${ }^{18-20}$ We imaged cleaved surface of a mica disc $(\phi=6 \mathrm{~mm}$, Furuuchi Chemical) by a cantilever with a spring constant of $\sim 42 \mathrm{~N} / \mathrm{m}(\mathrm{NCH}$, Nanoworld) in phosphate buffered saline (PBS) solution. For this experiment, we used a commercially available AFM controller (ARC2, Asylum Research) with a home-built analog feedback circuit. We also imaged cleaved surface of a calcite crystal $\left(5 \times 5 \mathrm{~mm}^{2}\right.$, Furuuchi Chemical) by a cantilever with a spring constant of $\sim 5 \mathrm{~N} / \mathrm{m}$ (UHF-1250, AppNano) in pure water. (Note that this cantilever has been discontinued and replaced by ACCESS-UHF.) For this experiment, we used a fully digital home-built AFM controller. In both the experiments, we regulated the tip-sample distance such that the

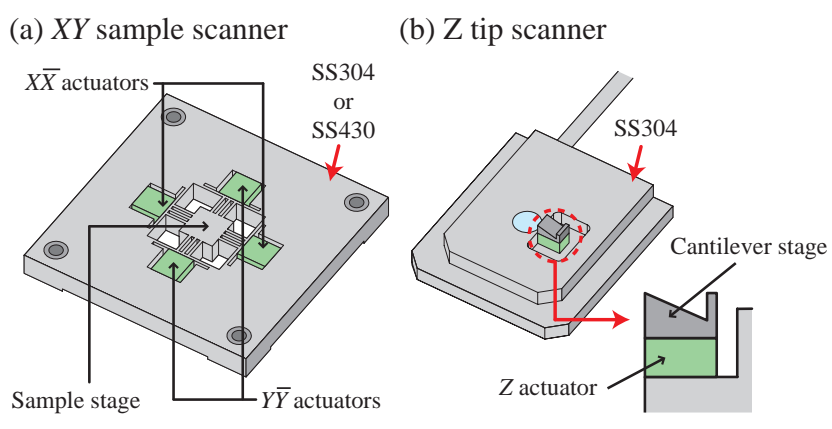

FIG. 1. Schematic models of the developed (a) $X Y$ and (b) $Z$ scanners.

averaged cantilever deflection equals zero to minimize the loading force.

\section{SEPARATE-TYPE HIGH-SPEED SCANNER}

\section{A. Basic design}

Figure 1 shows schematic model of the developed $X Y$ sample and $Z$ tip scanners. The separate-type design ensures independence of the two scanners. It also allows us to design simple scanners with a high resonance frequency. For the $X Y$ scanner [Fig. 1(a)], we used a flexure stage with four-fold symmetry, which is similar to the design reported by Hansma et al. ${ }^{9-13}$ The main body is made of stainless steel (SS304 or SS430). The sample stage at the center is connected to the four sides by three beams for each. A pair of stack piezoactuator (AE0203D04F, NEC Tokin) was used for driving each axis. For example, to drive the stage in $X$ direction, the $X$ and $\bar{X}$ actuators are extended and contracted, respectively. The use of two actuators improves the symmetry and linearity and thereby gives high stability and accuracy. In this design, there is no electric component below the sample stage, which helps us to avoid electric leakage through the spilled electrolyte solution.

The $Z$ scanner [Fig. 1(b)] consists of a multilayer piezoactuator (PL033, PI Ceramics) and a metal piece (cantilever stage) glued on it. The cantilever base is fixed on the cantilever stage with glue or other holding mechanisms as discussed in Sec. III C. Due to the simple structure and small size, it has high stability and resonance frequency. In addition, there are almost no low frequency spurious resonances. Although the electrodes of the actuator are not in contact with solution during AFM imaging, we sealed them with insoluble epoxy glue to prevent possible electric leakage.

\section{B. Sample holding mechanism}

\section{Basic design}

The sample holding mechanism is one of the key components that determines the usability and operation speed of a scanner. We have compared three types of sample holding mechanisms to optimize the design as shown in Fig. 2. We designed all the mechanisms to have a maximum sample size of $\sim 6 \mathrm{~mm}$. The first method is to glue a sample directly on the sample stage [Fig. 2(a)]. In this method, the sample is firmly 

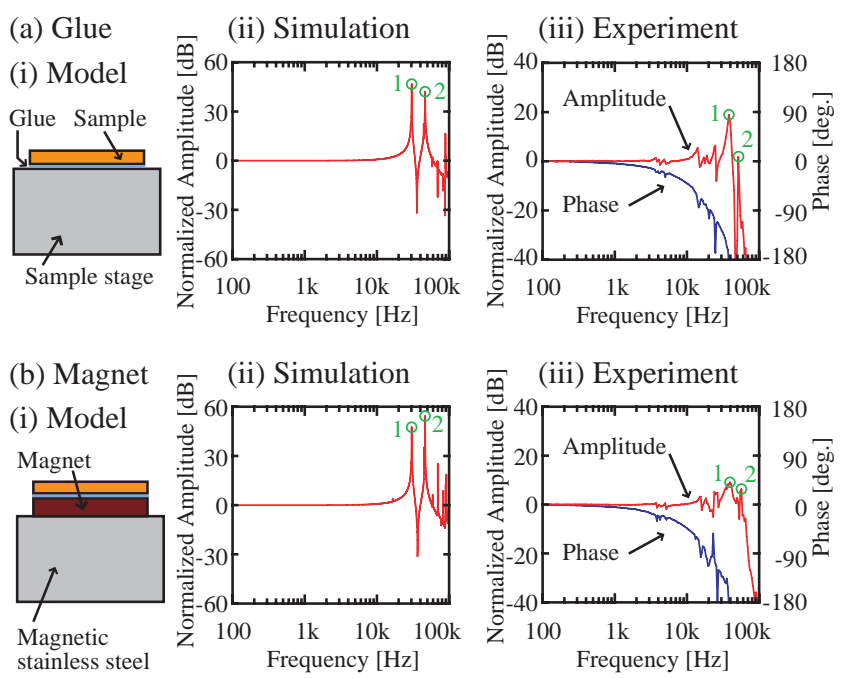

(c) Screw

(i) Model
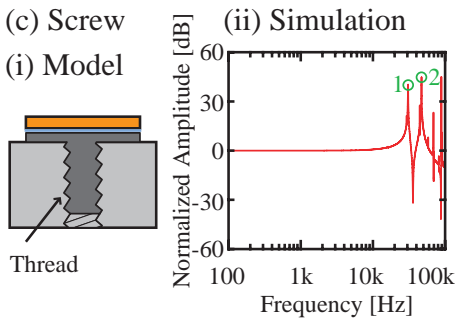

FIG. 2. Sample holding mechanisms using (a) glue, (b) magnet, and (c) screw. (i) Schematic models. (ii) Frequency response calculated by FEA. (iii) Frequency response measured by AFM.

fixed on the stage $\left(6 \times 6 \mathrm{~mm}^{2}\right)$ so that low frequency spurious resonances are not generated. However, replacement and reuse of a sample are very difficult. The second method is to fix a sample by magnetic force [Fig. 2(b)]. In this method, a sample is glued to a thin neodymium plate $(\phi=6 \mathrm{~mm})$. We made the scanner body of magnetic stainless steel (SS430) so that the plate is magnetically fixed to the sample stage. The third method is to fix a sample with a screw [Fig. 2(c)]. In the method, a sample is glued to a sample holder $(\phi=6 \mathrm{~mm})$ having a male thread. Then, the sample holder is screwed to the sample stage with a female thread.

\section{Frequency response}

Figure 2(ii) shows the frequency response of the $X Y$ scanners with different sample holding mechanisms calculated by FEA. All the curves show two peaks around 30 and $46 \mathrm{kHz}$, which correspond to Modes 1 and 2 shown in Fig. 3, respectively. In Mode 1, the scanner body is deformed in the same direction as the sample stage. On the contrary, in Mode 2, they are deformed in the directions opposite to each other.

The results suggest that the resonance frequency may be improved by increasing the thickness of the scanner body (3.5 $\mathrm{mm}$ in the present design). However, the increase of the thickness can limit the design flexibility of the whole AFM system. To gain an insight into this point, we calculated the lowest resonance frequency of the other XY scanners with a thickness of $7.5 \mathrm{~mm}$. If we increase the thickness of the whole part of the scanner including the flexure hinges and
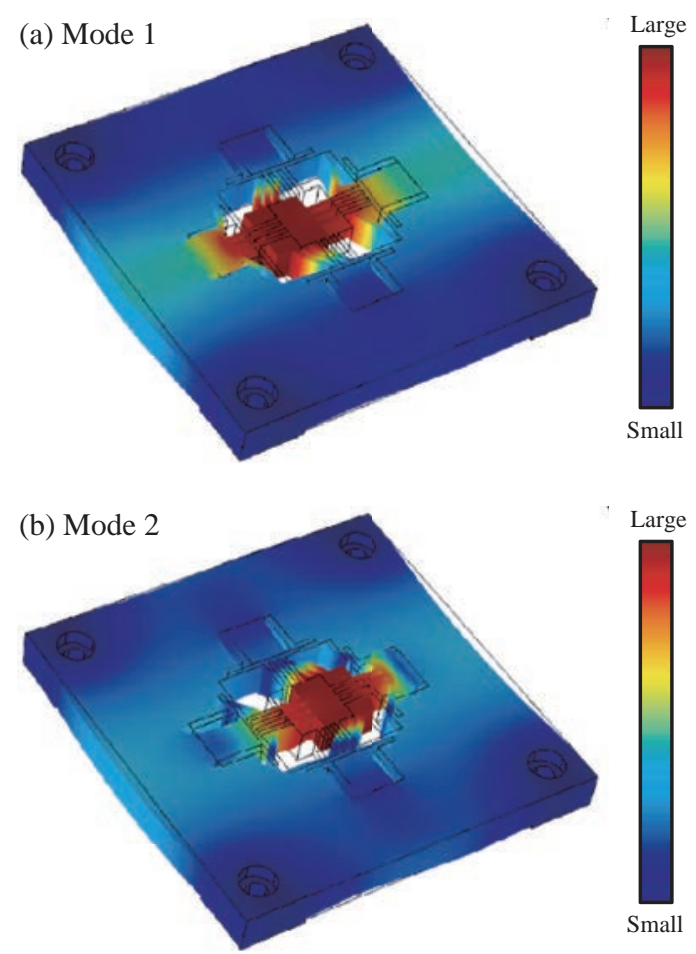

FIG. 3. Vibration modes of the $X Y$ scanner calculated by FEA. (a) Mode 1 and (b) Mode 2 corresponding to Peaks 1 and 2 in Fig. 2(ii), respectively.

stage, the resonance frequency decreases to $26.5 \mathrm{kHz}$ due to the increase of the mass. If we increase the thickness of the surrounding part only, the resonance frequency increases to $31.8 \mathrm{kHz}$. Even if we increase the thickness more than twice, the increase of the resonance frequency is only $5 \%$. Therefore, we have used the minimum thickness value (i.e., $3.5 \mathrm{~mm}$ ) that is compatible with the used piezoactuators to obtain the maximum flexibility of the AFM design. As the resonance frequency of $30 \mathrm{kHz}$ is acceptable for most of the high-speed AFM applications, we believe that the present design achieves a good balance between the usability and the performance.

Figure 2(iii) shows frequency response of the $X Y$ scanners measured by experiments. These curves show several peaks. Among them, two peaks around 40 and $55 \mathrm{kHz}$ are commonly observed in all the curves. Thus, we have attributed these peaks to the vibrations at Modes 1 and 2 shown in Fig. 3, respectively.

The curves also show minor peaks corresponding to the vibrations of spurious resonances. Among them, the low frequency peaks around $3 \mathrm{kHz}$ should determine the maximum drive frequency of the scanner. These peaks are commonly observed in all the curves and show similar profiles. Thus, the spurious resonances associated with these peaks are not likely to be in the holding mechanism but in the scanner body or the cantilever holder.

In spite of the influence of the spurious resonances, the amplitude and phase curves show almost flat response below $1 \mathrm{kHz}$. The result suggests that we can use all of the three holding mechanisms for scanning the stage up to $1 \mathrm{kHz}$. This $X$ scanning frequency is sufficiently fast for most of the highspeed AFM applications. 
In the magnetic holding mechanism, a sample can be easily fixed to the sample stage with an arbitrary rotation angle in $X Y$ plane. Its drawback is possible influence on the measurements of magnetic properties. It may also prevent other techniques using magnetic field. For example, in the magnetic cantilever excitation method, ${ }^{21}$ an ac magnetic field is applied to a cantilever, which may induce a vibration of the magnetic plate on the sample stage. In the screw holding mechanism, we can firmly fix a sample but its rotation angle cannot be controlled. In this case, we can use a non-magnetic material for the sample holder so that the method is compatible with other magnetic techniques.

In summary, both these two holding mechanisms are applicable to high-speed imaging. However, they have different advantages and disadvantages and hence are complimentary to each other. Therefore, we should choose one of them by taking into account the requirements in an application.

\section{Cantilever holding mechanism}

\section{Basic design}

We have compared three different cantilever holding mechanisms for the $Z$ tip scanner as shown in Fig. 4. In the glue holding mechanism [Fig. 4(a)], a cantilever is directly glued to the cantilever stage. Thus, the cantilever is tightly fixed to the holder and few spurious resonances are generated. However, this design makes it difficult to reuse the cantilever. In addition, exchanging cantilevers takes relatively long time as we need to wait for the glue to set.
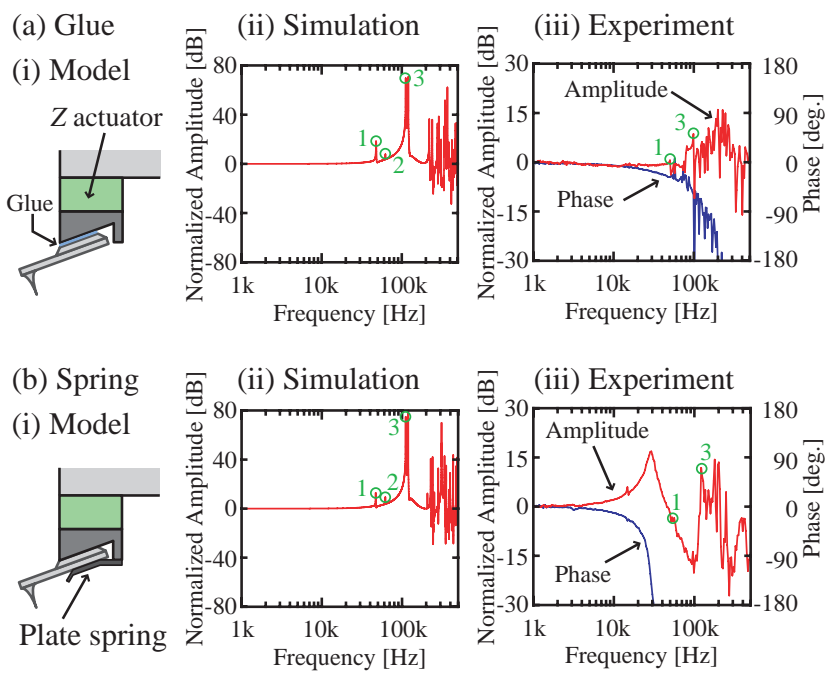

(c) Screw

(i) Model
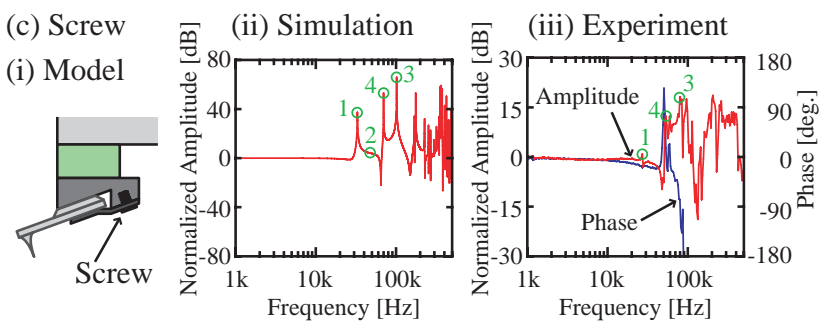

FIG. 4. Cantilever holding mechanisms using (a) glue, (b) plate spring, and (c) screw. (i) Schematic models. (ii) Frequency response calculated by FEA. (iii) Frequency response measured by an interferometer.
In the spring holding mechanism [Fig. 4(b)], a thin metal plate is fixed on the cantilever stage by spot welder. A cantilever is pushed down on the cantilever stage by the plate spring. In the screw holding mechanism [Fig. 4(c)], we use a cantilever stage with a female thread. A cantilever is sandwiched between the stage and a metal plate fixed with a screw.

\section{Frequency response}

Figure 4(ii) shows frequency response of the $Z$ scanners with different cantilever holding mechanisms calculated by FEA. The curve obtained with the glue holding mechanism shows two minor peaks (Peaks 1 and 2) around $50-70 \mathrm{kHz}$ and one major peak (Peak 3) at $110 \mathrm{kHz}$. According to the mode analysis shown in Fig. 5, Peaks 1 and 2 correspond to the swing (Mode 1) and twist (Mode 2) motion of the piezoactuator. Peak 3 corresponds to the normal vibration mode (Mode 3) of the free end of the cantilever base.

These three peaks are also found in the frequency response obtained with the spring holding mechanism [Fig. 4(b)]. However, the one obtained with the screw holding mechanism [Fig. 4(c)] shows a different profile with additional peaks. Based on the mode analysis, we have identified Peaks 1-4 to Modes 1-4 shown in Fig. 5, respectively. Note that Peak 2 is so small that it is hardly seen in Fig. 4. Peak 4 corresponds to the normal vibration mode of the protruded part of the cantilever stage. As this part does not exist in the glue and spring holding mechanisms, Peak 4 is found only in Fig. 4(c).

Figure 4(iii) shows experimentally measured frequency response curves. The amplitude curve obtained with the glue holding mechanism shows peaks at 50 and $100 \mathrm{kHz}$. By comparing these frequencies with those of the peaks found in the
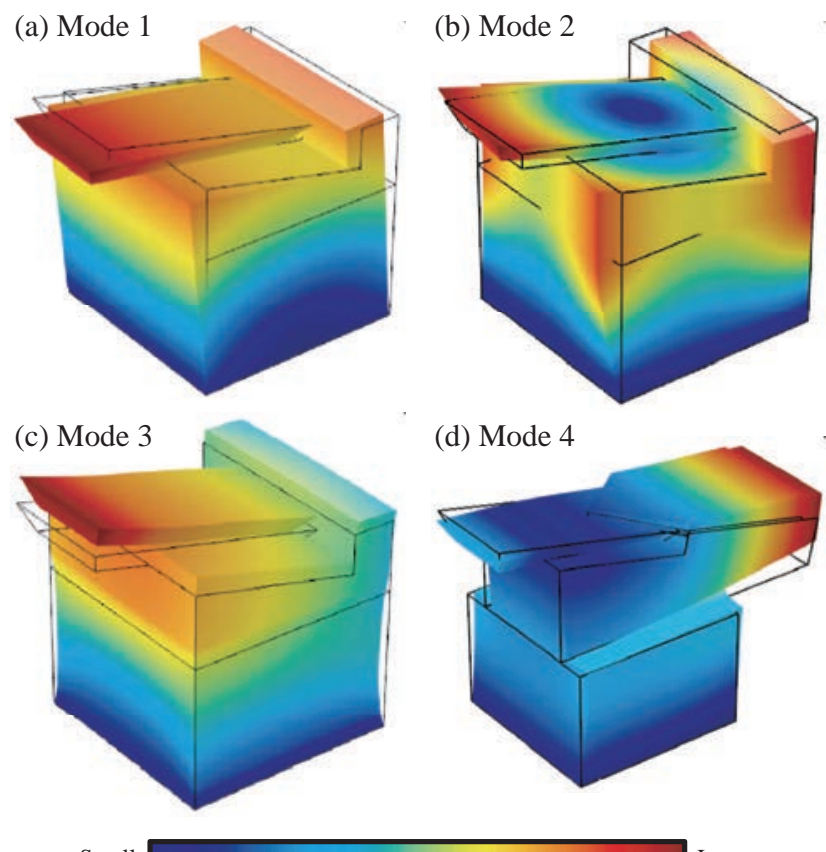

(d) Mode 4

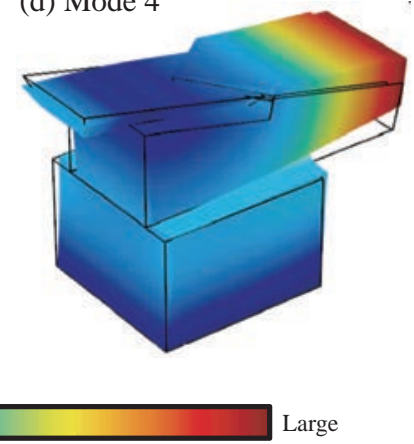

FIG. 5. Vibration modes of the $Z$ scanner calculated by FEA. Modes 1-4 correspond to Peaks 1-4 indicated in Fig. 4(ii), respectively. 
FEA curves, we have attributed them to Modes 1 and 3, respectively. A peak corresponding to Mode 2 is not found in the curve obtained by experiment. The FEA curves show that the peak corresponding to Mode 2 is very small. Thus, it was probably too small to be detected by experiment. The amplitude and phase curves show almost flat response below $10 \mathrm{kHz}$. This is sufficiently fast for most of the high-speed AFM applications. However, due to the use of glue, exchanging and reusing cantilevers are difficult.

The curve obtained with the spring holding mechanism shows a different profile from the one obtained by FEA. Although the peaks corresponding to Mode 1 (at $50 \mathrm{kHz}$ ) and Mode 3 (at $100 \mathrm{kHz}$ ) are visible in the both curves, a large peak at $30 \mathrm{kHz}$ does not exist in the curve obtained by FEA. We experimentally confirmed that even a subtle change in the holding force of the plate spring gives significant difference in the peak position and shape. Therefore, the peak is probably caused by the weak holding force of the plate spring. Although we tested several spring plates with different designs, it was impossible to eliminate this peak. Therefore, we have concluded that this design is not suitable for the highspeed AFM imaging.

The curve obtained with the screw holding mechanism shows a similar profile to the one obtained by FEA. Although the peak corresponding to Mode 2 is too small to be detected, other peaks corresponding to Modes 1,3 , and 4 are found in the both curves. The amplitude and phase curves show almost flat response up to $10 \mathrm{kHz}$. Thus, this design can be used for the high-speed AFM imaging. In addition, the design enables easy exchange and reuse of cantilevers. Therefore, the usability is much better than that of the glue holding mechanism.

\section{HIGH-VOLTAGE AMPLIFIER}

\section{A. Basic design}

In this study, we aim at developing a scanning system that enables high-speed and atomic-resolution imaging. Thus, the HVA should satisfy the requirements not only in the speed but also in the noise performance. To achieve this goal, we have developed wideband and low-noise high-voltage amplifiers specifically designed for driving the developed separatetype scanners.

Figure 6 shows schematic diagrams of the developed high-voltage amplifiers. One of the advantages of designing a dedicated amplifier is that we can assume exact values of the scanner specifications for the design. The specifications of the developed scanners and the piezoactuators used in them are listed in Table I.

In the $Z$ HVA, the input signal $(-10$ to $+10 \mathrm{~V})$ is attenuated by a factor of two $(-5$ to $+5 \mathrm{~V})$. The attenuated signal is added to a $+10 \mathrm{~V}$ reference by an inverting adder $(-5$ to $-15 \mathrm{~V})$. This signal is amplified by an inverting amplifier with a gain of -4 to obtain the $Z$ drive signal $(+20$ to $+60 \mathrm{~V})$.

In the $X Y$ scanner, a pair of piezoactuators used for driving one axis should be symmetrically driven. Thus, each of the $X$ and $Y$ HVAs should output two symmetrical signals (a) Driver for $Z$ piezoactuator

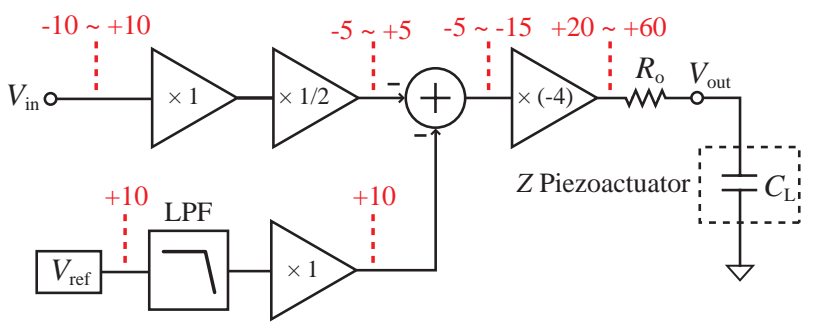

(b) Driver for $X$ piezoactuator

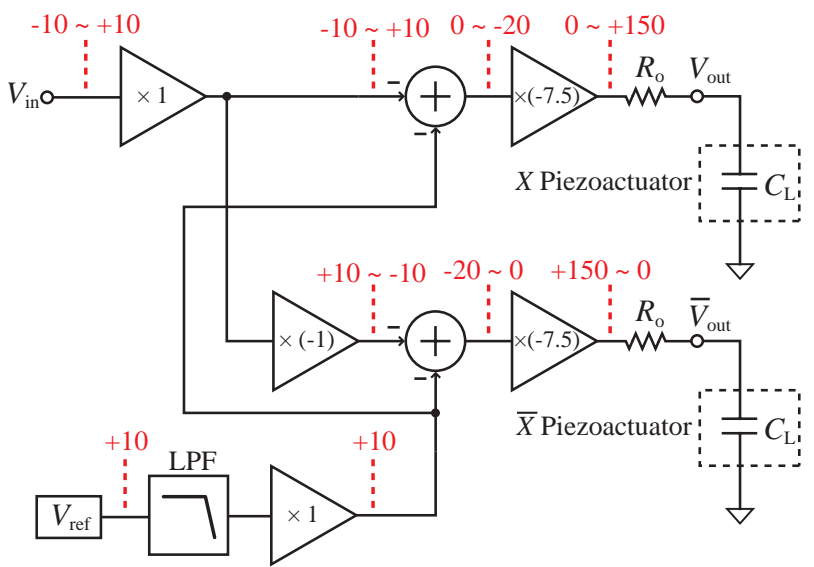

FIG. 6. Schematic diagrams of the developed high-voltage amplifiers for the (a) $Z$ and (b) $X$ scanners.

with an offset of $+75 \mathrm{~V}$. In the $X$ HVA, the input signal $(-10$ to $+10 \mathrm{~V})$ is added to $\mathrm{a}+10 \mathrm{~V}$ reference by an inverting adder $(0$ to $-20 \mathrm{~V})$. This signal is amplified by an inverting amplifier with a gain of -7.5 to obtain the $X$ driving signal $(0$ to $+150 \mathrm{~V})$. The other drive signal $(\bar{X}$ signal $)$ is produced through a similar circuit with an additional inverter. The design of the HVA for the $Y$ scanner is the same as that for the $X$ scanner.

To suppress a circuit oscillation caused by the large capacitance $\left(C_{\mathrm{L}}\right)$ of a piezoactuator, we used an output resistor $\left(R_{\mathrm{O}}\right)$. While the value of $R_{\mathrm{O}}$ should be high enough to suppress the oscillation, it should be kept as small as possible to achieve a wide bandwidth. We experimentally investigated the minimum $R_{\mathrm{O}}$ value required for suppressing the oscillation and found it to be $8.2 \Omega$.

Assuming that the bandwidth of the HVA $\left(B_{\mathrm{d}}\right)$ is determined by the cutoff frequency of a low-pass filter (LPF)

TABLE I. Specifications of the developed $Z$ and $X Y$ scanners and HVAs.

\begin{tabular}{llll}
\hline \hline & & \multicolumn{1}{c}{$Z$} & \multicolumn{1}{c}{$X Y$} \\
\hline Scanner & Sensitivity (nm/V) & 6.0 & 32.3 \\
& $C_{\mathrm{L}}(\mathrm{nF})$ & 90 & 75 \\
\multirow{4}{*}{ HVA } & Input range (V) & -10 to 10 & -10 to 10 \\
& Output range (V) & 20 to 60 & 0 to 150 \\
Scanning system & Gain & 2 & 7.5 \\
& Scan range (nm) & 240 & 4845 \\
& $B_{\mathrm{d}}(\mathrm{kHz})$ & 260 & 204 \\
\hline \hline
\end{tabular}


consisting of $R_{\mathrm{o}}$ and $C_{\mathrm{L}}, B_{\mathrm{d}}$ is given by $B_{\mathrm{d}}=1 /\left(2 \pi C_{\mathrm{L}} R_{\mathrm{o}}\right) . B_{\mathrm{d}}$ values are calculated by this equation and listed in Table I. For the both scanners, $B_{\mathrm{d}}$ is higher than $200 \mathrm{kHz}$. This is sufficiently fast for most of the high-speed AFM applications.

We used power supply voltages of $\pm 24 \mathrm{~V}$ instead of $\pm 15 \mathrm{~V}$ for the low-voltage operational amplifiers (OPA604, Texas Instruments). The $\pm 24 \mathrm{~V}$ power supply allows addition of the input $(-10$ to $+10 \mathrm{~V})$ and $+10 \mathrm{~V}$ reference signals without attenuation. This helps to keep the gain of the high-voltage amplifier as small as possible. The attenuation circuit in the $Z$ HVA is implemented to avoid driving the $Z$ actuator near $0 \mathrm{~V}$, where the nonlinearity of the actuator becomes prominent. For driving the high-voltage amplifiers (PA98, Cirrus Logic), we used power supply voltages of $-24 \mathrm{~V}$ and $+160 \mathrm{~V}$ instead of $\pm 160 \mathrm{~V}$. This is for keeping the heat generation as small as possible and eliminating the need for a cooling fan. We used a high precision voltage reference integrated circuit (AD587, Analog Devices) for generating the $+10 \mathrm{~V}$ reference. Although the output noise of an AD587 is relatively small, we found that it is not negligible. Thus, we used a LPF to reduce the noise to a negligible value.

\section{B. Frequency response}

Figure 7 shows frequency response of the developed HVAs measured with and without a capacitive load $\left(C_{\mathrm{L}}=100 \mathrm{nF}\right)$. The response curves obtained with the $Y$ HVA are similar to those obtained with the $X$ HVA and hence are omitted from the figure.

The gains of the $X$ and $Z$ HVAs at the low frequency range are 7.5 and 2 , respectively. These values agree with their design specifications. The amplitude curves show that the $-3 \mathrm{~dB}$ bandwidth measured without $C_{\mathrm{L}}$ is higher than $800 \mathrm{kHz}$ for both the HVAs. However, the insertion of a capacitive load $\left(C_{\mathrm{L}}=100 \mathrm{nF}\right)$ reduces the bandwidth to $399 \mathrm{kHz}$ and $289 \mathrm{kHz}$
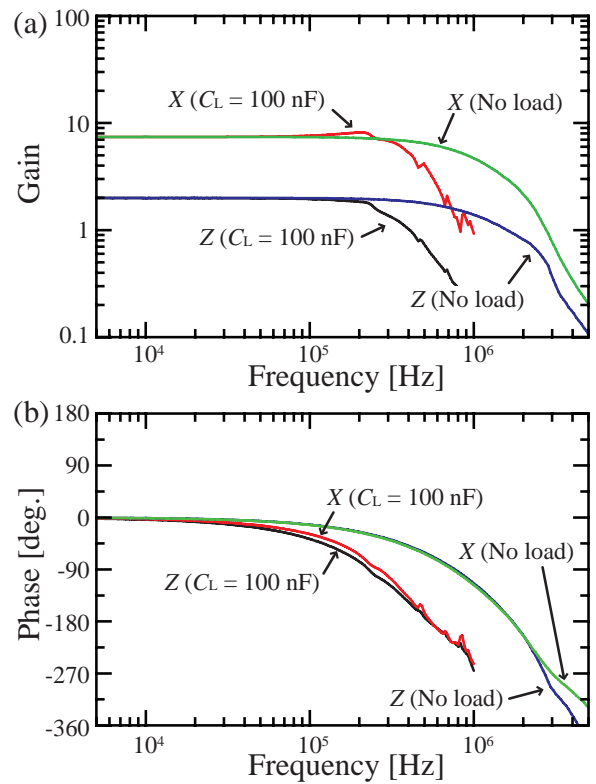

FIG. 7. Frequency response of the developed HVAs. (a) Amplitude curve. (b) Phase curve. for the $X$ and $Z$ HVAs, respectively. These values are slightly larger than the cut off frequency of the LPF consisting of $C_{\mathrm{L}}$ and $R_{\mathrm{o}}$ (i.e., $B_{\mathrm{d}}=194 \mathrm{kHz}$ ). This is because the gain damping is partially compensated by the small gain peak of the amplifier.

In this study, we aim at driving the $Z$ scanner at $10 \mathrm{kHz}$. Even with the capacitive load, the amplitude curves show almost flat response up to $100 \mathrm{kHz}$. The phase curves of the both HVAs show $4^{\circ}$ phase delay at $10 \mathrm{kHz}$. This is sufficiently small for achieving the $10 \mathrm{kHz}$ bandwidth in the tip-sample distance regulation. If necessary, further improvement may be achieved by reducing the capacitance of the $Z$ piezoactuator by sacrificing the scanning range.

\section{Noise performance}

Figure 8 shows output voltage spectral density distribution of the developed $X$ and $Z$ HVAs measured with and without a capacitive load. The curves measured without a capacitive load show a peak at 2-3 MHz. However, this peak is suppressed by the insertion of a capacitive load $\left(C_{\mathrm{L}}=100 \mathrm{nF}\right)$. We have also confirmed that the peak can be suppressed even with a smaller capacitive load $(\sim 10 \mathrm{nF})$. Thus, the gain peaking should not affect the AFM performance in the actual experiments.

The curve measured with a capacitive load shows that the roll-off frequency of the noise spectrum corresponds to the expected $B_{\mathrm{d}}$ value (i.e., $194 \mathrm{kHz}$ ). This result confirms that the bandwidth is limited by the LPF consisting of $R_{\mathrm{o}}$ and $C_{\mathrm{L}}$. At the frequency range less than $200 \mathrm{kHz}$, the curves show an almost constant value, namely, 396 and $196 \mathrm{nV} / \sqrt{\mathrm{Hz}}$ for the $X$ and $Z$ HVAs, respectively. Assuming that the bandwidth is $200 \mathrm{kHz}$, the displacement noises caused by the HVAs are calculated to be $5.7 \mathrm{pm}$ for the $X$ scanner and $0.53 \mathrm{pm}$ for the $Z$ scanner. In general, atomic-resolution AFM imaging requires spatial resolution of 100 and $10 \mathrm{pm}$ for $X Y$ and $Z$ axes, respectively. The estimated noises caused by the HVAs are negligible compared to these criteria. Thus, the HVAs should be applicable to atomic-resolution imaging.

For comparison, we also measured the performance of commercially available HVAs: HVA4-0222N (SPECS) and ENP-4014B (Echo Electronics) (Table II). Here we refer to these HVAs as HVA1 and HVA2, respectively. The HVA1 features low output noise and has been widely used for

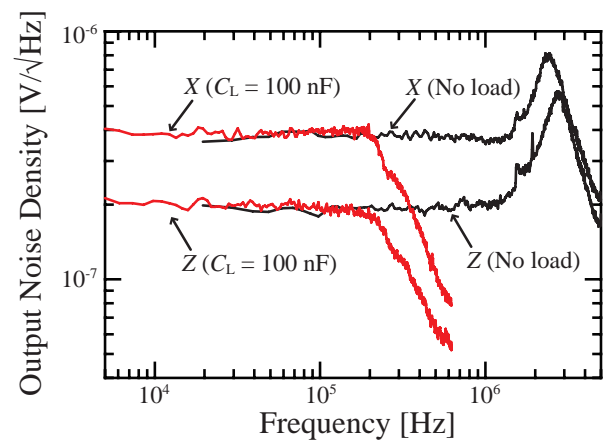

FIG. 8. Output voltage spectral density distribution of the developed $X$ and $Z$ HVAs measured with and without a capacitive load. 
TABLE II. Performance of the developed HVA and the commercially available ones.

\begin{tabular}{|c|c|c|c|c|}
\hline & \multirow[b]{2}{*}{ HVA1 } & \multirow[b]{2}{*}{ HVA2 } & \multicolumn{2}{|c|}{ Custom-built HVA } \\
\hline & & & $X Y$ & $Z$ \\
\hline Bandwidth $(\mathrm{kHz})$ & 4 & 150 & 200 & 200 \\
\hline Noise density $(\mathrm{nV} / \sqrt{\mathrm{Hz}})$ & 515 & 2685 & 396 & 196 \\
\hline
\end{tabular}

atomic-resolution AFM imaging. It showed $515 \mathrm{nV} / \sqrt{\mathrm{Hz}}$ noise and $4 \mathrm{kHz}$ bandwidth. Although the noise is sufficiently small, it cannot be used for high-speed imaging due to the narrow bandwidth. The HVA2 features wide bandwidth and has been used for various applications including scanning probe microscopy and magnetics research. It showed $2685 \mathrm{nV} / \sqrt{\mathrm{Hz}}$ noise and $150 \mathrm{kHz}$ bandwidth. Although the bandwidth is sufficiently wide, it cannot be used for atomic-resolution imaging due to the large noise.

Our custom-built HVAs achieve a lower noise than that of HVA1 and a wider bandwidth than that of HVA2. This is mainly because our HVA is specifically designed for the developed scanner with fixed gains and output ranges. On the contrary, the commercially available HVAs should be designed for a wide range of scanners with flexible gains and output ranges. Therefore, it is essential to develop dedicated HVAs for the atomic-resolution and high-speed AFM imaging.

\section{HIGH-SPEED AFM}

\section{A. Feedback bandwidth}

Figure 9 shows the frequency response of the tip-sample distance regulation measured with the developed scanners and HVAs. The measurement was performed in contact-mode AFM in liquid. We set the gains of the proportional-integral (PI) feedback controller such that the bandwidth is maximized.

If we define the feedback bandwidth $\left(B_{\mathrm{FB}}\right)$ as a frequency giving $-45^{\circ}$ phase delay, ${ }^{22} B_{\mathrm{FB}}$ is estimated to be $8 \mathrm{kHz}$ from the phase curve. The amplitude curve shows a peak at $12 \mathrm{kHz}$, which approximately agrees with the $B_{\mathrm{FB}}$ value estimated from the phase curve. The $8 \mathrm{kHz} B_{\mathrm{FB}}$ is sufficient for

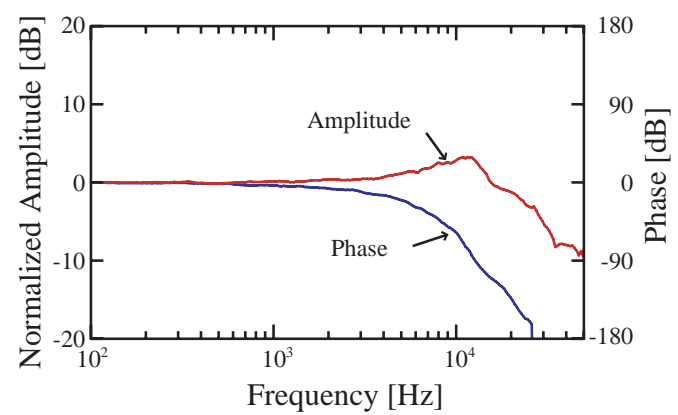

FIG. 9. Frequency response of the tip-sample distance feedback regulation measured with the developed scanners and HVAs. The measurement was performed in contact-mode AFM in liquid. imaging interfacial phenomena taking place at a time scale of a few seconds as shown in Secs. V B and V C.

\section{B. Imaging of mica}

Figure 10 shows AFM images of mica obtained in PBS solution using the developed scanning system. All the images were obtained with a scan rate of $78 \mathrm{~Hz}$ and a pixel resolution of $128 \times 64$. Thus, the tip velocity $\left(v_{\mathrm{t}}\right)$ increases with increasing the scan size. For Figs. 10(a) $-10(\mathrm{~d}), v_{\mathrm{t}}=0.39,0.59$, 1.17 , and $1.56 \mu \mathrm{m} / \mathrm{s}$, respectively. These scan speeds may appear to be slow compared to the values previously reported on large-scale and high-speed imaging. However, we should consider the number of corrugations that has to be visualized in one atomic-resolution image. For example, in Fig. 10(d), 370 atoms are visualized in one image. This number is much larger than that for a typical large-scale image. Therefore, we need to consider the detection frequency of the surface corrugations $\left(f_{\text {cr }}\right)$ rather than $v_{\mathrm{t}}$. For the cleaved mica surface, the atomic-scale corrugation period is $\sim 0.52 \mathrm{~nm}$. Thus, $f_{\text {cr }}$ is 0.75 , 1.13, 2.25, and $3 \mathrm{kHz}$ for Figs. 10(a)-10(d), respectively.

For quantitative estimation of the image corrugation, we calculated root-mean-square (RMS) amplitude of the each image. The RMS value was multiplied by $2 \sqrt{2}$ to obtain a peakto-peak corrugation $z_{\mathrm{pp}}$. For Figs. 10(a) $-10(\mathrm{~d}), z_{\mathrm{pp}}=3.93$, $3.79,3.38$, and $3.29 \mathrm{pm}$, respectively. The image obtained with the highest $f_{\mathrm{cr}}$ shows only $16 \%$ decrease in $z_{\mathrm{pp}}$ from the one obtained with the lowest $f_{\text {cr }}$. The result shows that the developed scanning system is capable of imaging atomic-scale structures with $3 \mathrm{kHz} B_{\mathrm{FB}}$.
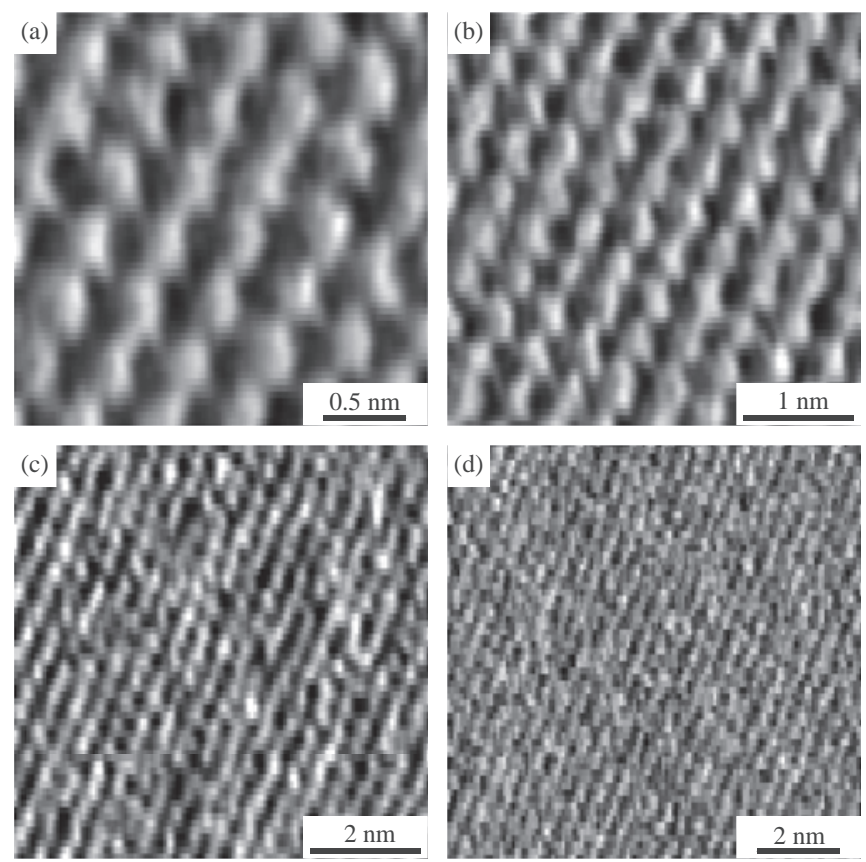

FIG. 10. Contact-mode AFM images of mica in PBS solution obtained with the developed scanners and HVAs. The screw holding mechanism was used for both the sample and cantilever holders. Scan rate: $78.125 \mathrm{~Hz}$. Imaging speed: $0.82 \mathrm{~s} /$ frame. Pixels: $128 \times 64$ pix. For (a)-(d), $v_{t}=0.39,0.59,1.17$, and $1.56 \mu \mathrm{m} / \mathrm{s}, f_{\mathrm{cr}}=0.75,1.13,2.25$, and $3 \mathrm{kHz}, z_{\mathrm{pp}}=3.93,3.79,3.38$, and $3.29 \mathrm{pm}$, respectively. 

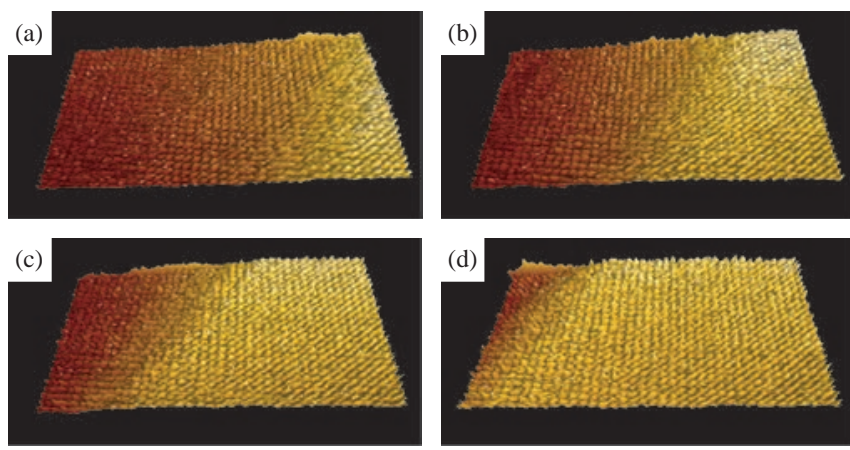

FIG. 11. Snapshots of 66 successive contact-mode AFM images of the calcite crystal growth process in water (see video in the supplementary material). ${ }^{17}$ (a) $0 \mathrm{~s}$. (b) $20 \mathrm{~s}$. (c) $40 \mathrm{~s}$. (d) $60 \mathrm{~s}$. The screw holding mechanism was used for both the sample and cantilever holders. Scan size: 20 $\times 10 \mathrm{~nm}^{2}$. Scan rate: $100 \mathrm{~Hz}$. Imaging speed: 2 s/frame. Pixel size: 200 $\times 200$ pix. Tip velocity: $4 \mu \mathrm{m} / \mathrm{s}$.

\section{Imaging of calcite}

Figure 11 shows snapshots of 66 successive contactmode AFM images of a cleaved calcite surface in water. A video file consisting of these AFM images is included in the supplementary material. ${ }^{17}$ Each image was taken in $2 \mathrm{~s}$ with a tip velocity of $4 \mu \mathrm{m} / \mathrm{s}$. Even with the fast scanning speed, individual atoms are clearly resolved in the images. The spacing between the atoms constituting a calcite surface is separated by approximately $0.5 \mathrm{~nm}$. Thus, the result shows that the scanning system allows us to obtain a clear atomic-scale image in liquid with $f_{\text {cr }}$ of $8 \mathrm{kHz}$. This high-speed and high-resolution imaging capability has enabled in situ imaging of the calcite crystal growth process with atomic-scale resolution.

The developed scanning system can be used for the realtime imaging of various solid/liquid interfacial phenomena with atomic-scale resolution. This capability should be particularly important for the studies on crystal growth, dissolution, and corrosion processes, where local atomic-scale arrangements play important roles.

\section{ACKNOWLEDGMENTS}

This work was supported by New Energy and Industrial Technology Development Organization (NEDO) (Grant No. 09A22005a) and ACT-C, Japan Science and Technology Agency.

${ }^{1}$ N. Kodera, D. Yamamoto, R. Ishikawa, and T. Ando, Nature (London) 468, 72 (2010).

${ }^{2}$ T. Uchihashi, R. Iino, T. Ando, and H. Noji, Science 333, 755 (2011).

${ }^{3}$ G. T. Paloczi, B. L. Smith, P. K. Hansma, D. A. Walters, and M. A. Wendman, Appl. Phys. Lett. 73, 1658 (1998).

${ }^{4}$ N. Sanz, J. K. Hobbs, and M. J. Miles, Langmuir 20, 5989 (2004).

${ }^{5}$ G. Binnig, C. F. Quate, and Ch. Gerber, Phys. Rev. Lett. 56, 930 (1986).

${ }^{6}$ T. Ando, N. Kodera, E. Takai, D. Maruyama, K. Saito, and A. Toda, Proc. Natl. Acad. Sci. U.S.A. 98, 12468 (2001).

${ }^{7}$ A. D. L. Humphris, M. J. Miles, and J. K. Hobbs, Appl. Phys. Lett. 86, 034106 (2005).

${ }^{8}$ T. Ando, T. Uchihashi, and T. Fukuma, Prog. Surf. Sci. 83, 337 (2008).

${ }^{9}$ J. H. Kindt, G. E. Fantner, J. A. Cutroni, and P. K. Hansma, Ultramicroscopy 100, 259 (2004).

${ }^{10}$ G. E. Fantner, G. Schitter, J. H. Kindt, T. Ivanov, K. Ivanova, R. Patel, N. Holten-Andresen, J. Adams, P. J. Thurner, I. W. Rangelow, and P. K. Hansma, Ultramicroscopy 106, 881 (2006).

${ }^{11}$ G. Schitter, P. J. Thurner, and P. K. Hansma, Mechatronics 18, 282 (2008).

${ }^{12}$ I. S. Bozchalooi, K. Youcef-Toumi, D. J. Burns, and G. E. Fantner, Rev. Sci. Instrum. 82, 113712 (2011).

${ }^{13}$ C. Braunsmann and T. E. Schäffer, Nanotechnology 21, 225705 (2010).

${ }^{14}$ F. C. Tabak, E. C. M. Disseldorp, G. H. Wortel, A. J. Katan, M. B. S. Hesselberth, T. H. Oosterkamp, J. W. M. Frenken, and W. M. van Spengen, Ultramicroscopy 110, 599 (2010).

${ }^{15}$ T. Fukuma, H. Okazaki, N. Kodera, T. Uchihashi, and T. Ando, Appl. Phys. Lett. 92, 243119 (2008).

${ }^{16}$ Y. K. Yong, S. O. R. Moheimani, B. J. Kenton, and K. K. Leang, Rev. Sci. Instrum. 83, 121101 (2012).

${ }^{17}$ See supplementary material at http://dx.doi.org/10.1063/1.4802262 for the video consisting of 66 successive AFM images showing the calcite crystal growth process and the COMSOL models of the $X Y$ and $Z$ scanners used for the FEA in this study.

${ }^{18}$ T. Fukuma, M. Kimura, K. Kobayashi, K. Matsushige, and H. Yamada, Rev. Sci. Instrum. 76, 053704 (2005).

${ }^{19}$ T. Fukuma and S. P. Jarvis, Rev. Sci. Instrum. 77, 043701 (2006).

${ }^{20}$ T. Fukuma, Rev. Sci. Instrum. 80, 023707 (2009).

${ }^{21}$ S. P. Jarvis, A. Oral, T. P. Weihs, and J. B. Pethica, Rev. Sci. Instrum. 64, 3515 (1993).

${ }^{22}$ T. Ando, T. Uchihashi, N. Kodera, A. Miyagi, R. Nakakita, H. Yamashita, and M. Sakashita, Jpn. J. Appl. Phys. 45, 1897 (2006). 